\title{
Ezetimibe, a Selective Inhibitor of the Transport of Cholesterol
}

\author{
Shin-ichiro Miura and Keijiro Saku
}

\begin{abstract}
Niemann-Pick C1-like 1 (NPC1L1) has recently been identified and has been shown to have features of a plasma membrane transporter, including a secretion signal, 13 predicted transmembrane domains, extensive $\mathrm{N}$-linked glycosylation sites and a sterol-sensing domain. It is highly expressed on the surface of absorptive jejunal enterocytes. NPC1L1 has been shown to be a direct target of ezetimibe, and an ezetimibe-sensitive pathway plays a role in intestinal cholesterol absorption. Ezetimibe-based therapy represents an exciting new area in the treatment of dyslipidemia.
\end{abstract}

Key words: Niemann-Pick C1-like 1, ezetimibe, cholesterol absorption

(Inter Med 47: 1165-1170, 2008)

(DOI: 10.2169/internalmedicine.47.1099)

\section{Introduction}

Dietary and biliary cholesterol are taken up by intestinal epithelial cells and transported to the endoplasmic reticulum (1). Pharmacological intervention through the inhibition of intestinal cholesterol absorption is a useful strategy for treating patients with dyslipidemia. Ezetimibe [1-(4fluorophenyl)-(3R)-[3-(4-fluorophenyl)-(3S)-hydroxy propyl](4S)-(4-hydroxypropyl)-2-azetidinone] potently inhibits cholesterol and phytosterol uptake (2). Niemann-Pick C1-like 1 (NPC1L1) has recently been identified and has been shown to have features of a plasma membrane transporter, including a secretion signal, 13 predicted transmembrane domains, extensive N-linked glycosylation sites and a sterol-sensing domain (3) (Fig. 1). It is highly expressed on the surface of absorptive jejunal enterocytes. NPC1L1 has been shown to be a direct target of ezetimibe, and an ezetimibe-sensitive pathway plays a role in intestinal cholesterol absorption (4). These findings represent an exciting new area in the treatment of dyslipidemia. This review will address the roles of NPC1L1 and its inhibitor ezetimibe in cardiovascular disease.

\section{Molecular Basis of Niemann-Pick C1-Like 1 (NPC1L1)}

The two known complementation groups of NiemannPick disease type C (NPC), NPC1 and NPC2, result from non-allelic protein defects. NPC1 disease caused by defects in the NPC1 protein is a recessive cholesterol-storage disorder characterized by severe, progressive neurodegeneration (5). NPC1L1 is a $145-\mathrm{kDa}$ membrane protein (6). NPC1L1 is mapped to chromosome $7 \mathrm{p} 13$, contains 20 exons, including an unusually large 1526-bp exon 2 , and spans approximately $29 \mathrm{~kb}$ (7). NPC1L1 exists in the putative promoter region that contains a sterol-regulatory element. The predicted protein shared $42 \%$ identity and $51 \%$ similarity with NPC1. NPC1L1 contains a conserved amino-terminal NPC1 domain and a putative sterol-sensing domain (Fig. 1). Altmann et al reported that NPC1L1 is mainly expressed in the small intestine and in the brush border membrane of enterocytes, and humans highly express NPC1L1 in the liver (3, 7). NPC1L1 exists as a predominantly cell-surface membrane-expressed protein, consistent with its proposed role as a putative intestinal sterol transporter (6).

NPC1L1-deficient mice show a substantial reduction in absorbed cholesterol. Based on data from NPC1L1-deficient mice, about $70 \%$ of cholesterol absorption in the intestine depends on NPC1L1. The remaining 30\% is independent of

Department of Cardiology, Fukuoka University School of Medicine, Fukuoka Correspondence to Dr. Keijiro Saku, saku-k@ cis.fukuoka-u.ac.jp 


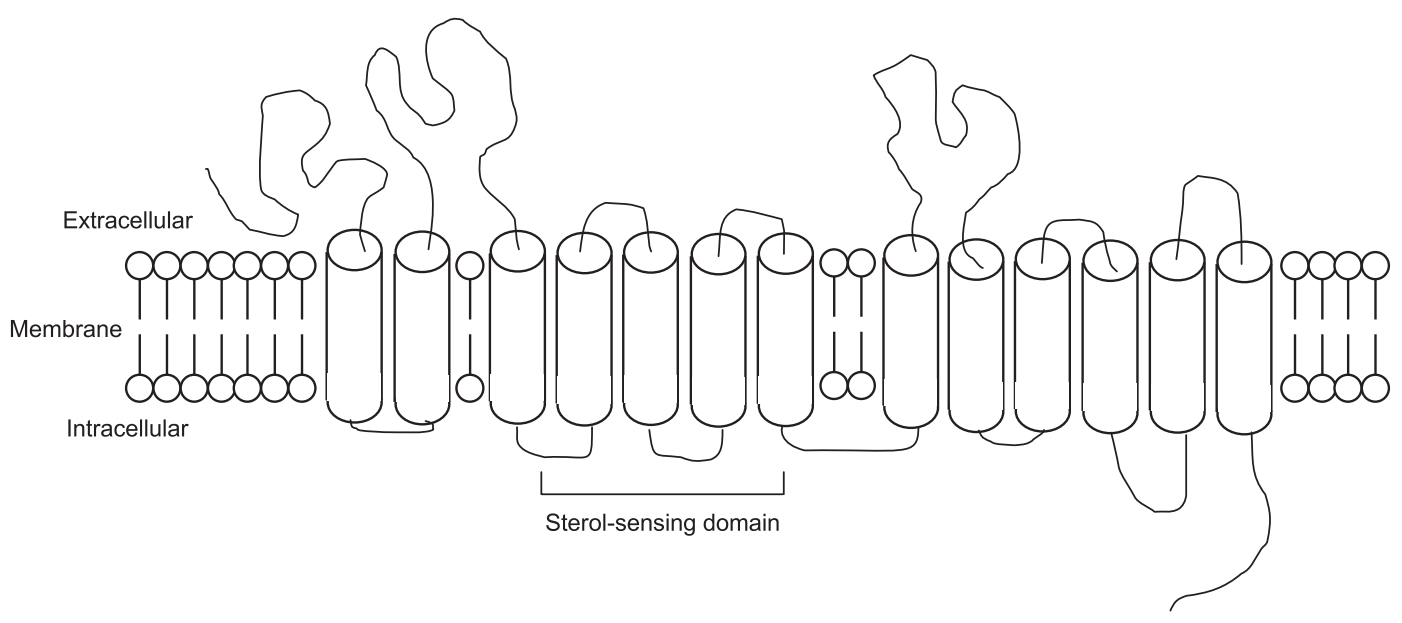

Figure 1. Secondary structure of NPC1L1.

NPC1L1, and may be through scavenger receptor type BI (SR-BI) (8) or other transporters. However, it is still unclear whether NPC1L1 is a high-affinity cholesterol-binding protein on intestinal brush border membranes. Deficiencies in either NPC1L1 or PNC1 L2 protein do not affect cholesterol uptake or absorption (9). SR-BI, not NPC1L1, is the major protein that is responsible for the initial high-affinity cholesterol ligand binding process in the cholesterol absorption pathway. Additionally, ezetimibe may inhibit brush border membrane cholesterol binding through targets such as SR-BI in addition to its inhibitory effects on NPC1L1, based on the results in NPC1L1-deficient mice (10).

Although the regulation of NPC1L1 expression has not been completely defined, the level of NPC1L1 expression can be altered by various treatments or conditions. NPC1L1 mRNA expression is downregulated in wild-type and NPC1 L1-deficient mice fed a cholesterol/cholate diet (11). NPC1L1 mRNA levels are decreased in vivo, in the duodenum of mice treated with the liver $\mathrm{X}$ receptor (LXR) agonist T0901317 (12). Human NPC1L1, as well as human NPC1, can functionally substitute for the Caenorhabditis elegans genes ncr-1 and/or ncr-2, which are known to play a role in the process of dauer formation (a process which can be modulated by cholesterol in sensitized genetic backgrounds) (13). More recently, Tang et al reported that NPC1L1 is required for a liver $\mathrm{x}$ receptor (LXR) agonist to increase plasma high-density lipoprotein (HDL) cholesterol in mice (14). NPC1L1 mRNA levels are significantly decreased in the small intestine of phospholipid transfer protein (PLTP) KO mice (15). Peroxisome proliferator-activated receptor- $\delta$ (PPAR- $\delta$ ) activation decreases NPC1L1 mRNA levels and cholesterol trafficking to the endoplasmic reticulum. PPAR- $\delta$ activation is associated with increased plasma HDL and reduced intestinal cholesterol absorption efficiency that may be related to the decreased intestinal NPC1L1 expression (16). In diabetic animals, NPC1L1 mRNA is significantly elevated and ATP-binding cassette transporter (ABC) G5 and ABCG8 are significantly reduced in the liver (17). Pioglitazone significantly reduces NPC1L1 mRNA and significantly increases ABCG5 and G8 mRNA compared to insulin (17). The expression of ABCG5 and ABCG8 mRNA is lower in diabetic patients and microsomal triglyceride transfer protein (MTTP) expression is increased (18) (Fig. 2). The enhanced NPC1L1 expression is correlated with cholesterol depletion, consistent with transcriptional regulation.

\section{Molecular Target of Ezetimibe}

Ezetimibe blocks the uptake of oxidized low-density lipoprotein (LDL) in human macrophages (19). This compound has no effect on the uptake and/or processing of acetylated LDL (Ac-LDL). In addition, it blocks the lipid-induced induction of the LXR/RXR target genes ABCA1, ABCG1, and apolipoprotein $\mathrm{E}$ more effectively in macrophages loaded with Ox-LDL than in those loaded with Ac-LDL. Fenofibrate affects the ezetimibe-sensitive pathway and retains the ability to decrease cholesterol absorption and NPC1L1 mRNA expression in chow-fed LXR alpha/beta-doubleknockout mice and high-cholesterol- or cholic acid-fed wildtype mice. Fenofibrate may act via peroxisome proliferatoractivated receptor $\alpha($ PPAR $\alpha)$ to decrease cholesterol absorption at the level of intestinal NPC1L1 expression (20). In an in vivo study, Temel et al reported that in transgenic mice expressing human NPC1L1 in hepatocytes, hepatic overexpression of NPC1L1 induces a decrease in biliary cholesterol, but not phospholipid or bile acid concentrations, in association with a 30-60\% increase in plasma cholesterol (21). Since these effects are blocked by treatment with ezetimibe, ezetimibe may reduce plasma cholesterol by inhibiting NPC1L1 function in both the intestine and liver (21). In addition, a direct interaction between NPC1L1 and ezetimibe was shown in an in vitro binding assay (22). Ezetimibe failed to bind to membranes collected from NPC1 L1-deficient mice compared to wild-type mice, which indicates that ezetimibe interacts directly with NPC1L1. Hepatic NPC1L1 is also important for regulating biliary cholesterol excretion and is a target of ezetimibe, and may protect the body from excessive biliary loss of cholesterol. Moreover, 
A.

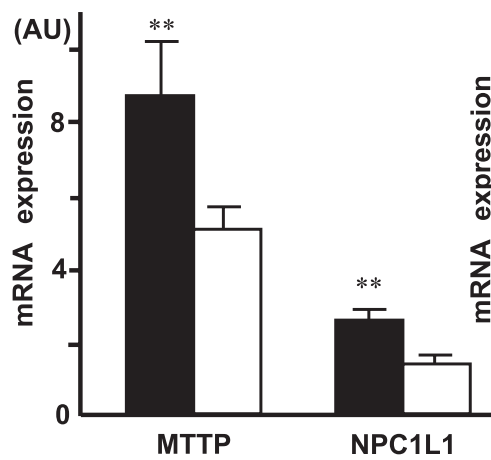

B.

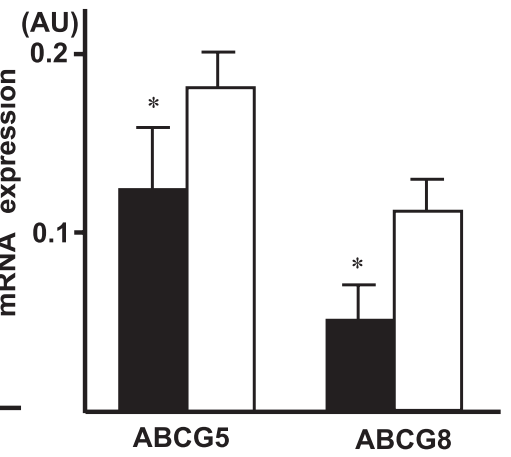

Figure 2. Comparison of messenger (m)RNA expression of genes (A) microsomal triglyceride transfer protein (MTTP) and Niemann-Pick C1-like 1 (NPC1L1), (B) ATP-binding cassette transporter (ABC) $\mathrm{G} 5$ and $\mathrm{ABCG8}$ in the dysregulation of postprandial lipoproteins in type 2 diabetes (black bars) and non-diabetic control patients (white bars). Mean $\pm \mathrm{SE}$. *p< $<0.05$, **p $<0.02$ compared to control subjects.

ezetimibe improves the insulin and plasma glucose response after intraperitoneal glucose injection, and enhances insulin signaling in cultured hepatocytes (23).

\section{Beneficial Effects of Ezetimibe in Humans}

\section{Monotherapy}

Ezetimibe can effectively lower plasma LDL cholesterol to decrease cholesterol absorption (24). Ezetimibe $10 \mathrm{mg}$ / day induces approximately a $20 \%$ reduction in LDL cholesterol (25) (Fig. 3). In addition, ezetimibe decreases triglyceride (TG) by about $8 \%$ and increases high-density lipoprotein (HDL) cholesterol by about $5 \%$. In patients with primary dyslipidemias, ezetimibe $(10 \mathrm{mg} /$ day $)$ therapy for 16 weeks reduced total, LDL cholesterol and non-HDL cholesterol values as well as apolipoprotein B concentrations (26).

\section{Combination therapy}

\subsection{Ezetimibe/statin therapy}

Despite the availability of statins, many patients do not achieve lipid targets. Combination therapy with lipidlowering agents that act via a complementary pathway may allow additional patients to achieve their recommended cholesterol goals. Therefore, the combination of ezetimibe and statin may synergistically decrease cholesterol absorption and synthesis. The study group of Gagné et al included 769 adults with primary hypercholesterolemia who had not achieved National Cholesterol Education Program (NCEP) Adult Treatment Panel II goals with dietary alteration and statin monotherapy (27). Ongoing statin therapy plus ezetimibe led to significant changes of $-25.1 \%$ in LDL cholesterol (HDL cholesterol $+2.7 \%$; TG $-14.0 \%$ ) compared to LDL cholesterol $-3.7 \%$, HDL cholesterol $+1.0 \%$, and TG$2.9 \%$ with placebo (Fig. 4). Among patients who were not at the LDL cholesterol goal at on-statin baseline, $71.5 \%$ of

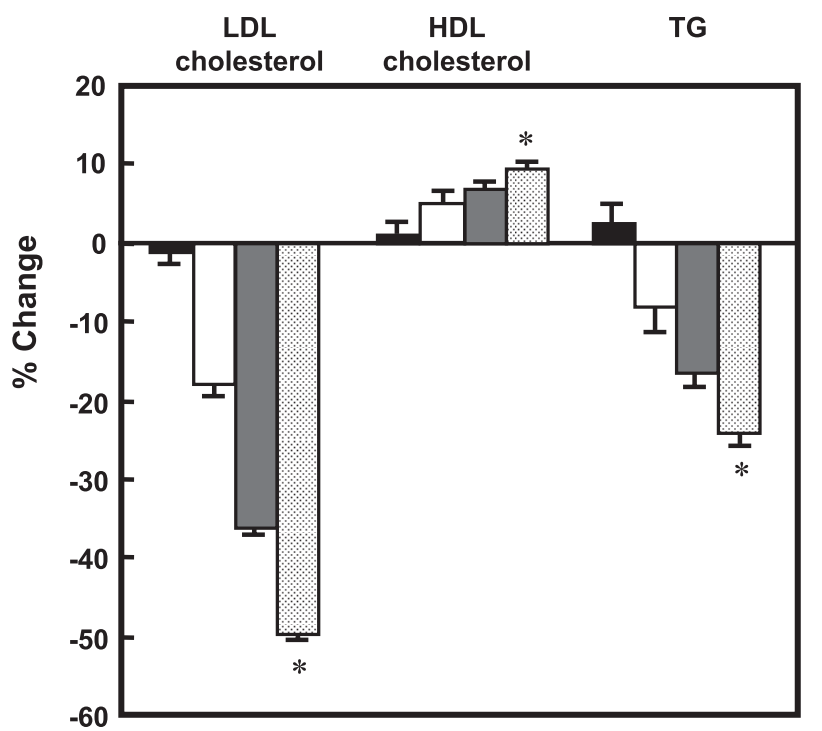

Figure 3. Changes in serum lipid profile in placebo (black bars), ezetimibe therapy (white bars), simvastatin therapy (gray bars), and ezetimibe+simvastatin therapy (dotted bars) in patients with primary hypercholesterolemia. LDL, lowdensity lipoprotein; HDL, high-density lipoprotein; TG, triglyceride. ${ }^{*} \mathbf{p}<0.05$ vs. simvastatin therapy.

those receiving statin plus ezetimibe versus $18.9 \%$ of those receiving statin plus placebo reached this goal at the endpoint. In multicenter, randomized, double-blind, placebocontrolled, $2 \times 5$ factorial studies, ezetimibe plus simvastatin significantly improved LDL cholesterol, HDL cholesterol, and TG compared to simvastatin alone. Ezetimibe plus simvastatin provided an incremental $13.8 \%$ LDL cholesterol reduction, 2.4\% HDL cholesterol increase, and 7.5\% TG reduction compared with pooled simvastatin alone (Fig. 3) (25). In patients with type 2 diabetes mellitus (DM), combination therapy is usually required to optimize glucose metabolism as well as to help patients achieve aggressive tar- 


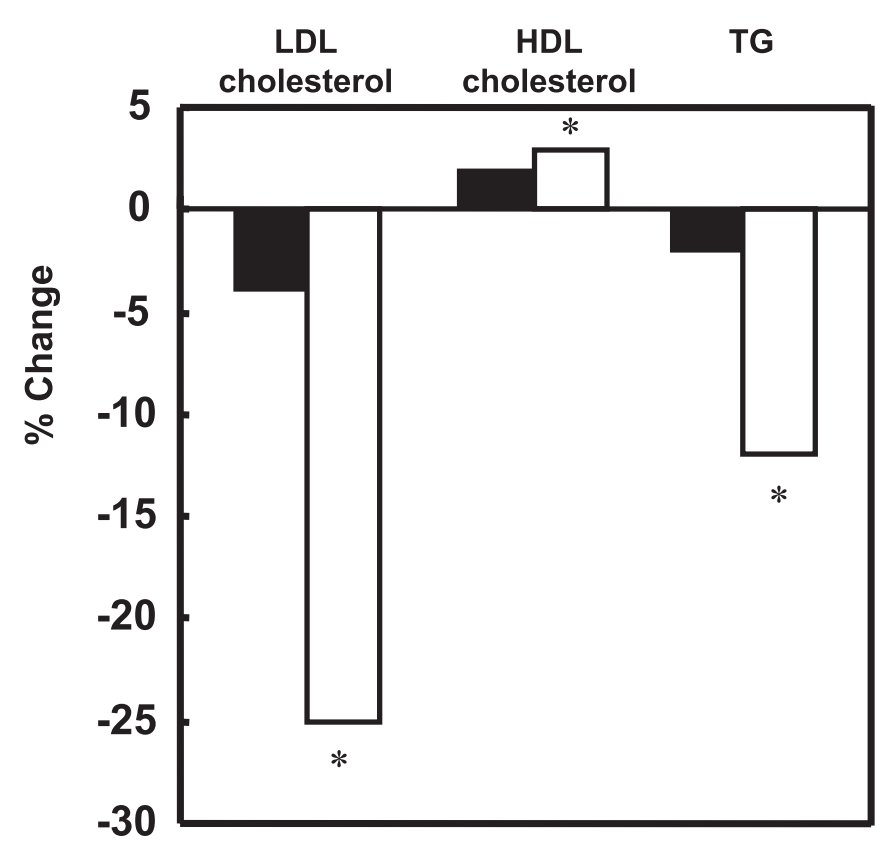

Figure 4. Ezetimibe added to ongoing statin therapy for the treatment of primary hypercholesterolemia. Black and white bars indicate statin monotherapy and ezetimibe+simvastatin combination therapy, respectively. LDL, low-density lipoprotein; HDL, high-density lipoprotein; TG, triglyceride. *p $<0.05$ vs. statin mono-therapy.

gets for LDL cholesterol and other lipid parameters associated with cardiovascular risk. A randomized, double-blind, parallel group, multicenter study in type 2 DM patients showed that LDL cholesterol was reduced significantly more by adding ezetimibe $10 \mathrm{mg} /$ day to simvastatin $20 \mathrm{mg} /$ day than by doubling the dose of simvastatin to $40 \mathrm{mg} /$ day (28). In addition, ezetimibe plus simvastatin $20 \mathrm{mg} /$ day also produced significant incremental reductions in non-HDL cholesterol, very low-density lipoprotein cholesterol and apolipoprotein B relative to simvastatin $40 \mathrm{mg} / \mathrm{day}$. In a double-blind study, 628 patients with baseline LDL cholesterol 145 to $250 \mathrm{mg} / \mathrm{dL}$ and $\mathrm{TG}<$ or $=350 \mathrm{mg} / \mathrm{dL}$ were randomly assigned to receive one of the following treatments for 12 weeks: ezetimibe (10 mg/day); atorvastatin $(10,20,40$, or $80 \mathrm{mg} /$ day $)$; ezetimibe $(10 \mathrm{mg}$ /day) plus atorvastatin $(10,20,40$, or $80 \mathrm{mg} /$ day); or placebo (29). Ezetimibe plus atorvastatin significantly improved LDL cholesterol, HDL cholesterol, TG and high-sensitivity C-reactive protein (hs-CRP) compared to atorvastatin alone. The addition of ezetimibe to on-going simvastatin treatment also resulted in a significantly greater mean percent reduction in LDL cholesterol from baseline (25.2\%) compared to with placebo $(0.9 \%)$. The EXPLORER study was designed to investigate the efficacy and safety of rosuvastatin $40 \mathrm{mg}$ alone or in combination with ezetimibe $10 \mathrm{mg} /$ day in patients at high risk of coronary heart disease (30). The combination of rosuvastatin/ezetimibe reduced LDL cholesterol significantly more than rosuvastatin. Other components of the lipid/lipoprotein profile were also significantly improved with rosu- vastatin/ezetimibe.

In familial hypercholesterolemia $(\mathrm{FH})$ patients, the combination of ezetimibe and statin therapy reduced LDL cholesterol by $57 \%$, regardless of the type of LDL receptor mutation. In clinical trials, ezetimibe plus simvastatin produced greater LDL cholesterol reductions than did monotherapy. A possible molecular mechanism of the enhanced efficacy of ezetimibe plus simvastatin is decreased VLDL and LDL apoB-100 concentrations through reduced VLDL production and the upregulation of LDLR-mediated LDL clearance (31). LDL cholesterol reduction in FH heterozygotes following combined therapy may affect the complex interplay between hepatic synthesis and the intestinal absorption of cholesterol.

Endothelium-dependent, acetylcholine-mediated vasodilation was significantly better with combination therapy. More effective restoration of endothelial function with a combination of statin and ezetimibe compared to statin monotherapy was seen in patients with metabolic syndrome (MetS). DM patients had more NPC1L1 mRNA than control subjects (18). Ezetimibe may be useful for the treatment of patients with DM and MetS in whom cholesterol absorption may be upregulated.

\subsection{Ezetimibe/fibrate or Ezetimibe/statin/fibrate ther- apy}

Both ezetimibe and fenofibrate offer complementary benefits to the lipid profile in patients with mixed hyperlipidemia. Patients in the fenofibrate plus ezetimibe and fenofibrate groups continued on their respective base study treatment, and patients in the ezetimibe and placebo groups were switched to fenofibrate plus ezetimibe and fenofibrate, respectively (32). Fenofibrate plus ezetimibe achieved significantly greater reductions in LDL cholesterol than fenofibrate alone. There were also significantly greater improvements in TG, HDL cholesterol, total cholesterol, non-HDL cholesterol, and apolipoprotein $\mathrm{B}$ with fenofibrate plus ezetimibe than with fenofibrate alone. In a multicenter, randomized, double-blind, placebo-controlled, parallel arm trial, the LDL cholesterol level was significantly reduced with ezetimibe/ simvastatin + fenofibrate compared with fenofibrate or placebo, but not when compared with ezetimibe/simvastatin (33). HDL cholesterol and apolipoprotein A-I levels were significantly increased with ezetimibe/simvastatin + fenofibrate treatment compared with ezetimibe/simvastatin or placebo. TG, non-HDL cholesterol, and apolipoprotein B levels were significantly reduced with ezetimibe/simvastatin + fenofibrate versus all other treatments. Thus, the coadministration of ezetimibe/simvastatin + fenofibrate effectively improved the overall atherogenic lipid profile of patients with mixed hyperlipidemia.

\section{Ezetimibe therapy on human genetic studies}

Naturally occurring coding mutations in the NPC1L1 gene might affect the response to ezetimibe. For example, different NPC1L1 protein variants $\left(\mathrm{Val}^{55}\right.$ to $\mathrm{Lue}^{55}$ and $\mathrm{Ile}^{1233}$ 
to $\mathrm{Asp}^{1233}$ ) have been found in a non-responder to ezetimibe (34). In addition, 3 common single-nucleotide polymorphisms have been found in NPC1L1 (1735C > G, 25342A > $\mathrm{C}$ and $27677 \mathrm{~T}>\mathrm{C}$ ). A total of 101 subjects were treated with ezetimibe for primary hypercholesterolemia. These patients showed a significant greater reduction in plasma LDL cholesterol with ezetimibe than patients with at least one copy of the haplotype $(-35.9+4.0 \%$ versus $-23.6+1.6 \%$ reduction). Characterization of DNA variations in NPC1L1 demonstrated that common variants in this gene are significantly associated with the LDL cholesterol levels response to treatment with ezetimibe/statin (35). Although rare variants identified in low-absorbers were found in AfricanAmericans and were associated with lower plasma levels of LDL cholesterol (36), none of these common or rare variants have been confirmed. However, evidence has indicated an association between the LDL cholesterol concentration and NPC1L1 gene production as a target for ezetimibe in humans.

\section{Safety of Ezetimibe in Humans}

Ezetimibe/statin combination therapy was well-tolerated in 2,382 patients with primary hypercholesterolemia (37). Moreover, the safety and tolerability profiles for the ezetimibe/simvastatin and ezetimibe monotherapy groups were similar in a randomized, double-blind, placebocontrolled trial (38). The combination therapy of ezetimibe and statin was equally safe across specific age groups: age < 65 versus more than 65 years; age $<75$ versus more than 75 years (39). Simvastatin therapy is a well-tolerated and effective therapy for children with heterozygous $\mathrm{FH}$ (40). In this way, ezetimibe shows a favorable safety profile across all patients.

\section{Conclusions}

NPC1L1 has been identified as a key player in cholesterol absorption, and is a useful target of cholesterol-lowering medication. Ezetimibe is one of the most important medications for inhibiting cholesterol absorption through NPC1L1. This compound has been shown to significantly improve lipid parameters as either monotherapy or in combination therapy with other lipid-lowering medications. Moreover, it has been shown to have a favorable safety characterization in all patients. In the future, large randomized trials should be performed to evaluate the effectiveness of ezetimibe/statin combination therapy versus statin monotherapy for the risk factors of cardiovascular disease.

\section{References}

1. Grundy SM. Absorption and metabolism of dietary cholesterol. Annu Rev Nutr 3: 71-96, 1983.

2. Clader JW. The discovery of ezetimibe: a view from outside the receptor. J Med Chem 47: 1-9, 2004.

3. Altmann SW, Davis HR Jr, Zhu LJ, et al. Niemann-Pick C1 Like 1 protein is critical for intestinal cholesterol absorption. Science 303: 1201-1204, 2004.

4. Garcia-Calvo M, Lisnock J, Bull $\mathrm{HG}$, et al. The target of ezetimibe is Niemann-Pick C1-Like 1 (NPC1L1). Proc Natl Acad Sci U S A 102: 8132-8137, 2005.

5. Ioannou YA. The structure and function of the Niemann-Pick C1 protein. Mol Genet Metab 71: 175-181, 2000.

6. Iyer SP, Yao X, Crona JH, et al. Characterization of the putative native and recombinant rat sterol transporter Niemann-Pick $\mathrm{C} 1$ Like 1 (NPC1L1) protein. Biochim Biophys Acta 1722: 282-292, 2005.

7. Davies JP, Levy B, Ioannou YA. Evidence for a Niemann-pick C (NPC) gene family: identification and characterization of NPC1L 1. Genomics 65: 137-145, 2000.

8. Hauser H, Dyer JH, Nandy A, et al. Identification of a receptor mediating absorption of dietary cholesterol in the intestine. Biochemistry 37: 17843-17850, 1998.

9. Dixit SS, Sleat DE, Stock AM, et al. Do mammalian NPC1 and NPC2 play a role in intestinal cholesterol absorption? Biochem J 408: 1-5, 2007.

10. Labonté ED, Howles PN, Granholm NA, et al. Class B type I scavenger receptor is responsible for the high affinity cholesterol binding activity of intestinal brush border membrane vesicles. Biochim Biophys Acta 1771: 1132-1139, 2007.

11. Davis HR Jr, Zhu LJ, Hoos LM, et al. Niemann-Pick C1 Like 1 (NPC1L1) is the intestinal phytosterol and cholesterol transporter and a key modulator of whole-body cholesterol homeostasis. J Biol Chem 279: 33586-33592, 2004.
12. Duval C, Touche V, Tailleux A, et al. Niemann-Pick $C 1$ like 1 gene expression is down-regulated by LXR activators in the intestine. Biochem Biophys Res Commun 340: 1259-1263, 2006.

13. Smith MM, Levitan DJ. Human NPC1L1 and NPC1 can functionally substitute for the ncr genes to promote reproductive development in C. elegans. Biochim Biophys Acta 1770: 1345-1351, 2007.

14. Tang W, Ma Y, Jia L, Ioannou YA, Davies JP, Yu L. Niemann-Pick C1-Like 1 is required for an LXR agonist to raise plasma HDL cholesterol in mice. Arterioscler Thromb Vasc Biol 28: 448-454, 2008.

15. Liu R, Iqbal J, Yeang $C$, et al. Phospholipid transfer proteindeficient mice absorb less cholesterol. Arterioscler Thromb Vasc Biol 27: 2014-2021, 2007.

16. van der Veen JN, Kruit JK, Havinga R, et al. Reduced cholesterol absorption upon PPARdelta activation coincides with decreased intestinal expression of NPC1L1. J Lipid Res 46: 526-534, 2005.

17. Lally S, Owens D, Tomkin GH. The different effect of pioglitazone as compared to insulin on expression of hepatic and intestinal genes regulating post-prandial lipoproteins in diabetes. Atherosclerosis 193: 343-351, 2007.

18. Lally S, Tan CY, Owens D, et al. Messenger RNA levels of genes involved in dysregulation of postprandial lipoproteins in type 2 diabetes: the role of Niemann-Pick C1-like 1, ATP-binding cassette, transporters G5 and G8, and of microsomal triglyceride transfer protein. Diabetologia 49: 1008-1016, 2006.

19. Seedorf U, Engel T, Lueken A, et al. Cholesterol absorption inhibitor Ezetimibe blocks uptake of oxidized LDL in human macrophages. Biochem Biophys Res Commun 320: 1337-1341, 2004.

20. Valasek MA, Clarke SL, Repa JJ. Fenofibrate reduces intestinal cholesterol absorption via PPAR \{alpha\}-dependent modulation of NPC1L1 expression in mouse. J Lipid Res 48: 2725-2735, 2007. 
21. Temel RE, Tang W, Ma Y, et al. Hepatic Niemann-Pick C1-like 1 regulates biliary cholesterol concentration and is a target of ezetimibe. J Clin Invest 117: 1968-1978, 2007.

22. Garcia-Calvo M, Lisnock J, Bull $\mathrm{HG}$, et al. The target of ezetimibe is Niemann-Pick C1-Like 1 (NPC1L1). Proc Natl Acad Sci U S A 102: 8132-8137, 2005.

23. Deushi M, Nomura M, Kawakami A, et al. Ezetimibe improves liver steatosis and insulin resistance in obese rat model of metabolic syndrome. FEBS Lett 581: 5664-5670, 2007.

24. van Heek M, Farley C, Compton DS, et al. Comparison of the activity and disposition of the novel cholesterol absorption inhibitor, SCH58235, and its glucuronide, SCH60663. Br J Pharmacol 129: 1748-1754, 2000.

25. Davidson MH, McGarry T, Bettis R, et al. Ezetimibe coadministered with simvastatin in patients with primary hypercholesterolemia. J Am Coll Cardiol 40: 2125-2134, 2002.

26. Kalogirou M, Tsimihodimos V, Gazi I, et al. Effect of ezetimibe monotherapy on the concentration of lipoprotein subfractions in patients with primary dyslipidaemia. Curr Med Res Opin 23: 1169-1176, 2007.

27. Gagné C, Bays HE, Weiss SR, et al. Efficacy and safety of ezetimibe added to ongoing statin therapy for treatment of patients with primary hypercholesterolemia. Am J Cardiol 90: 1084-1091, 2002.

28. Gaudiani LM, Lewin A, Meneghini L, et al. Efficacy and safety of ezetimibe co-administered with simvastatin in thiazolidinedionetreated type 2 diabetic patients. Diabetes Obes Metab 7: 88-97, 2005.

29. Ballantyne CM, Houri J, Notarbartolo A, et al. Effect of ezetimibe coadministered with atorvastatin in 628 patients with primary hypercholesterolemia: a prospective, randomized, double-blind trial. Circulation 107: 2409-2415, 2003.

30. Ballantyne CM, Weiss R, Moccetti T, et al. Efficacy and safety of rosuvastatin $40 \mathrm{mg}$ alone or in combination with ezetimibe in patients at high risk of cardiovascular disease (results from the EXPLORER study). Am J Cardiol 99: 673-680, 2007.
31. Telford DE, Sutherland BG, Edwards JY, et al. The molecular mechanisms underlying the reduction of LDL apoB-100 by ezetimibe plus simvastatin. J Lipid Res 48: 699-708, 2007.

32. McKenney JM, Farnier M, Lo KW, et al. Safety and efficacy of long-term co-administration of fenofibrate and ezetimibe in patients with mixed hyperlipidemia. J Am Coll Cardiol 47: 15841587, 2006.

33. Farnier M, Roth E, Gil-Extremera B, et al. Efficacy and safety of the coadministration of ezetimibe/simvastatin with fenofibrate in patients with mixed hyperlipidemia. Am Heart J 153 (335): e1-e8, 2007.

34. Wang J, Williams CM, Hegele RA. Compound heterozygosity for two non-synonymous polymorphisms in NPC1L1 in a nonresponder to ezetimibe. Clin Genet 67: 175-177, 2005.

35. Simon JS, Karnoub MC, Devlin DJ, et al. Sequence variation in NPC1L1 and association with improved LDL-cholesterol lowering in response to ezetimibe treatment. Genomics 86: 648-656, 2005.

36. Cohen JC, Pertsemlidis A, Fahmi S, et al. Multiple rare variants in NPC1L1 associated with reduced sterol absorption and plasma low-density lipoprotein levels. Proc Natl Acad Sci U S A 103: 1810-1805, 2006.

37. Davidson MH, Ballantyne CM, Kerzner B, et al. Efficacy and safety of ezetimibe coadministered with statins: randomised, placebo-controlled, blinded experience in 2382 patients with primary hypercholesterolemia. Int J Clin Pract 58: 746-755, 2004.

38. Goldberg AC, Sapre A, Liu J, et al. Efficacy and safety of ezetimibe coadministered with simvastatin in patients with primary hypercholesterolemia: a randomized, double-blind, placebocontrolled trial. Mayo Clin Proc 79: 620-629, 2004.

39. Lipka L, Sager P, Strony J, et al. Efficacy and safety of coadministration of ezetimibe and statins in elderly patients with primary hypercholesterolaemia. Drugs Aging 21: 1025-1032, 2004.

40. de Jongh S, Ose L, Szamosi T, et al. Efficacy and safety of statin therapy in children with familial hypercholesterolemia: a randomized, double-blind, placebo-controlled trial with simvastatin. Circulation 106: 2231-2237, 2002.

(C) 2008 The Japanese Society of Internal Medicine http://www.naika.or.jp/imindex.html 